\title{
The Association of Cachexia with Head and Neck Cancer Burden and Pathologic Features
}

\author{
Marelle Rukes ${ }^{1}$, Alexander Jones ${ }^{1,2}$, Leah Novinger ${ }^{1,2}$, Kyle Davis ${ }^{1}$, Vincent Campiti ${ }^{1}$, Grace \\ Yedlicka ${ }^{1}$, Andrea Bonetto ${ }^{1,2,3}$ \\ ${ }^{1}$ Indiana University School of Medicine; ${ }^{2}$ Department of Otolaryngology - Head \& Neck Surgery; \\ ${ }^{3}$ Department of Surgery
}

Background and Hypothesis: Head and neck cancer (HNC) is frequently associated with cachexia, characterized by involuntary weight loss, sarcopenia, and malnutrition. In HNC patients, dysphagia and anorexia from obstructive aerodigestive tumors propagates cachexia even further. However, the impact of pathologic features and burden of HNC on cachexia has yet to be investigated. We therefore hypothesize that larger, more aggressive tumors impose greater cachexia severity in HNC patients.

Methods: A single-institution, retrospective study of adult patients undergoing surgical resection of head and neck carcinoma from 2014-2017 was performed. Patients without 30-day preoperative abdominal CT imaging for skeletal muscle index (SMI, $\mathrm{cm}^{2} / \mathrm{m}^{2}$ ) measurements were excluded. Patient demographics, comorbidities, nutrition data, and cancer pathology reports were collected. Cachexia was defined as unintentional weight loss $>5 \%$ over 6 months or $>2 \%$ with $\mathrm{BMI}<20 \mathrm{~kg} / \mathrm{m}^{2}$. Statistical analyses were performed using 2-sided one-way Welch's ANOVA or Pearson's $\chi^{2}$ tests. Significance was determined at $p<0.05$.

Results: The cohort included 125 predominantly white (92.0\%), male (75.2\%) HNC patients age $59.9 \pm 11.5$ years. Sixty-seven (53.6\%) patients had cachexia, twenty $(16.0 \%)$ of whom were severe (weight loss $\geq 15 \%)$. Patients with severe cachexia had larger tumors $(5.5 \pm 2.1 \mathrm{~cm}$, $p=0.021$ ) than patients with mild-to-moderate cachexia (weight loss $5-14.9 \% ; 4.9 \pm 2.1 \mathrm{~cm}$ ) or no cachexia $(4.1 \pm 1.9 \mathrm{~cm})$. Worsening cachexia severity was also associated with lower SMI $(p=0.004)$, BMI $(p=0.002)$, and serum albumin $(p=0.011)$. There was no statistically significant difference between cachexia groups comparing patient age, comorbidities, tumor grade, depth of invasion, nodal metastases, cancer stage, perineural invasion, lymphovascular invasion, or extranodal extension.

Conclusion and Potential Impact: Tumor burden of HNC patients, but not adverse pathologic features, is associated with greater cachexia severity. Identifying pro-cachectic markers produced by larger tumors could provide a molecular target for anti-cachexia therapies and improve cancer patient outcomes. 